\title{
Editorial
}

\section{Creating a research agenda}

Journal of Revenue and Pricing Management (2017) 16, 343-344. doi:10.1057/s41272-017-0102-4

The Journal of Revenue and Pricing Management is at the forefront of research in revenue management (RM), the journal serves as a bridge between practice and theory because both are seen as of equal importance, as it is theory that informs practice and practice that shapes theory. Thus, creating a research agenda is about linkage between the two. Therefore, creating a research agenda is the aim of this journal and the platform for contributors within the field. Hence, what is a potential research agenda?

\section{AGENDA 1: VALUE PRICING}

To the consumer, RM has always been about value or the best price. However, with its movement shifting away from traditional systems, the concept of value is contested based upon how consumers differentiate and extract value. Therefore, there are gaps between intended and perceived differentiations. As value-based pricing within the context of the low-cost carrier is based more on price sensitivity and behavioural economics, the next generation of RM algorithms has yet to emerge.

\section{AGENDA 2: CODE SHARING}

Code share flights are flight segments that are operated by one carrier but marketing and sales performed by one or more carriers. It provides an airline with the capability to increase their marketing reach beyond limits established through bilateral agreements without investing in additional aircraft, crew, and ground resour- ces. However, there are lots of issues with availability status (AVS). To overcome the inherent limitations of AVS for O\&D carriers, the preferred method is bid price exchange. The research question is how do carriers determine the numeric availability for the true O\&D that includes code share segments?

\section{AGENDA 3: ORIGIN \& DESTINATION (O\&D)}

Network RM is one of the key future enhancements for airline RM. However, there are a number of challenges. Enhancing RM tools and availability, calculations from leg to O\&D and setting up the seamless links with all the relevant GDSs call for a heavy investment and increase operating costs issues that include the substantial investment in hardware and IT infrastructure to forecast the many thousands of O\&Ds at the lowest level of detail. Basically, network RM systems still have to find optimal solutions through network revenue maximisation. Every optimisation model requires simplifying assumptions and capturing the facets of RM variables; this is a real challenge for $\mathrm{RM}$ researchers.

\section{AGENDA 4: SECONDARY PRICING}

With the growth of ancillary sales such as luggage allowances, meals, travel insurance and sightseeing trips, secondary pricing is an area of growing importance in RM. The integration of such sales and RM systems is a key area of 
focus of RM researchers. One avenue of research is the development of total RM systems which entails the creation of dynamic customised, personalised offers based upon consumer traits, value scores, itineraries and availability of products and services.

\section{AGENDA 5: PRICING STRATEGIES}

In general, in academic terms, pricing has evolved into a fruitful area of research in terms of consumer behaviour, psychology and revenue management. The key question is, if pricing is so important to the firm, how does the organisation address capability that is beyond a model of 'hunch'.

\section{AGENDA 6: CONSUMER BEHAVIOUR}

The Internet has changed how consumers purchase products and experiences but the arrival of the Internet has brought about a series of unknowns in terms of consumer behaviour and RM. These questions involve correlation between online variable pricing practices and product offering.

\section{AGENDA 7: COMPETITIVE DATA}

The Internet era has made pricing transparent to the consumer resulting in increased price sensitivity, mercurial consumption and the consumer being a lot more savvy with regard to airline and hospitality pricing. Therefore, there is a growing recognition that the present RM systems that rely on historical booking and ticketing data and that do not provide inventory-control mechanisms in real time, which correlate to competitive data are becoming defunct. Thus, the future research agenda is Dynamic Availability (DA) overlaid with large data based on consumer attribute. However, what is unclear, is how we go about this.

Ian Yeoman

Victoria University of Wellington, Wellington,

New Zealand

E-mail: ian.yeoman@vuw.ac.nz 